\title{
The Primal and Dual Problems of b-Complementary Multisemigroup.
}

\author{
Eleazar Madriz \\ Universidade Federal do Recôncavo da Bahia \\ Cruz da Almas, BA, Brazil, \\ egmlozada@gmail.com
}

May 12, 2014

\begin{abstract}
In this work we present the primal and dual problem for a b-complementary multisemigroup.
\end{abstract}

\section{Introduction}

In general, for the master additive system problem (see [1],[2]) there exits results of lifting facets that can't be used for non-master because when projecting a facet of a master polyhedra onto a non-master polyhedra we don't obtain a facet of the non-master. In the case where the algebraic structure is an abelian group, Gastou (see [3]) showed how to lift in a sequential way. This way does not consider the dual problem of the problem associated in order to caracterize the facets, this is a motive for the research. The main purpose of this paper is to define the dual problem of a primal problem for a b-complementary multisemigroup. We have extended the result in [5] the semigroup for the bcomplementary multisemigroup.

\section{The b-complementary Multisemigroup.}

An additive system $(A, \widehat{+})$ is defined to be a non-empty finite set $A$ together with addition $\widehat{+}: 2^{A} \times 2^{A} \rightarrow 2^{A}\left(2^{A}=\{H: H \subset A\}\right)$ such that:

(1) $\{g\} \widehat{+}\{h\} \subseteq A$, for all $g$ and $h$ in $A$;

(2) $S \widehat{+} T=\cup_{s \in S, t \in T}(\{s\} \widehat{+}\{t\})$, for all $S, T \subseteq A$.

In this work we use $g \widehat{+} h$ in the place of $\{g\} \widehat{+}\{h\}$.

An identity is an element of $A$ denoted $\widehat{0}$ such that $\widehat{0} \widehat{+} g=g \widehat{+} \widehat{0}$, for all $g \in A$, a infinity, $\infty$, is the element of $A$ such that $\infty \widehat{+} S=S \widehat{+} \infty=\infty$, for all $S \in 2^{A}$. 
We assume that both identity and infinity, are unique if they exist. The set $A_{+}$ of proper elements is the set $A \backslash\{\widehat{0}, \infty\}$. In this work we denote $\mathrm{N}$ by the positive integer set and $\mathrm{R}$ as the set of real numbers.

An additive system is associative if :

$$
(S \widehat{+} T) \hat{+} U=S \widehat{+}(T \widehat{+} U)
$$

for all $S, T, U \in 2^{A}$. And, an additive system is abelian if $S \hat{+} T=T \widehat{+} S$, for all $S, T \in 2^{A}$.

An expression of the additive system $(A, \widehat{+})$ is defined recursively as follows $($ see $[4])$ :

(i) $(\xi)$ is an empty expression;

(ii) $(g)$ is a primitive expression, for all $g \in A$;

(iii) $E=\left(E_{1} \widehat{+} E_{2}\right)$ is an expression, for all expressions $E_{1}$ and $E_{2}$,

For $E$ as $\left(\right.$ iii) we call the expressions $E_{1}$ and $E_{2}$ the subexpression of $E$.

The evaluation $\gamma$ (see [4]) is the function of the expressions of $(A, \widehat{+})$ in the $2^{A}$ defined recursively by

- If $E=(\xi)$ then $\gamma(E)=\{\widehat{0}\}$;

- If $E=(g)$ then $\gamma(E)=\{g\}$, for all $g \in A$;

- If $E=\left(E_{1} \widehat{+} E_{2}\right)$ then $\gamma(E)=\gamma\left(E_{1}\right) \widehat{+} \gamma\left(E_{2}\right)$.

The incident vector of an expression $E$ is the vector $t=\left(t(g) ; g \in A_{+}\right)$such that $t(g)$ is the number of times that $(g)$ appears as the primitive subexpression of $E$. Now, let $b$ be a fixed element in an additive system $(A, \widehat{+})$, the expression $E$ is a solution expression of $b$ if $b \in \gamma(E)$. And $t \in \mathbf{N}^{A_{+}}$is a solution vector of $b$ if there are a solution expression $E$ of $b$.

Let $(A, \widehat{+})$ be an abelian associativity additive system. Since $(A, \widehat{+})$ is associative and abelian, for any positive integer $k$ and any $g \in A$, we can defined $k g$ by

$$
k g=\gamma((\overbrace{(g) \hat{+} . . \widehat{+}(g)}^{k-\text { times }})) .
$$

Now, since there are only a finite number of subsets of $A$ in the sequence of sets $0 g, 1 g, 2 g, \ldots, k g, \ldots$ there are sets which appear an infinite number of times, such sets are called loop sets of $g$. The loop of $g$ is the union of all the loop sets of $g$. Let $s=m g$ be the first occurrence of any set appearing for the second time in the sequence $(k g \mid k \geq 0)$. Since $s$ appears the second time in the sequence, $s=p g$ for some $p<m$, and the sequence of distinct sets $(k g \mid p<k \leq m-1)$ is the same as $(k g \mid m \leq k \leq 2 m-p-1)$. In fact we have $(p+k+i l) g=(p+k g)$ (where $l=m-p)$ for $0 \leq k \leq l-1$ and $i \geq 0$, since $(m+k) g=m g \hat{+} k g=p g \widehat{+} k g=(p+k) g$. The loop order of $g$ is defined to be this $l$. Clearly $h \in A$ is in loop of $g$ if and only if there exists $k \geq 0$ such that $h \in(k+i l) g$ for all $i \geq 0$, where $l$ is the loop order of $g$. 
Let $(A, \widehat{+})$ be an abelian associative additive system and $b \in A_{+}$. We say that $(A, \widehat{+})$ is $b-$ consistent, if and only if $b \in b \widehat{+} k g$, for all $g \in A$ and for all $k \in Z_{+}$, and that $(A, \widehat{+})$ is a multisemigroup if it's $g$-consistent for all $g \in A$.

Let $(A, \widehat{+})$ be an additive system and $b \in A$, we define

$$
b \sim g=\{x \in A: b \in x \hat{+} g\} .
$$

These sets induce a partial order in $A$, we say $g \widetilde{<} h$ when $b \sim g \subseteq b \sim h$. When the set $b \sim g$ has a minimum element, this minimum element is called $b$ - complement of $g$. The and is denoted by $\widehat{g}$. A additive system $A$ is called $b$-complementary when every element has a $b$-complement. An element $g \in A$ is infeasible whenever there is not solution of the equation $b \in g \hat{+} x$, that is , $b \sim g=\emptyset$. We can assume, without loss of generality, that the additive system has at most one infeasible element denoted by $\widehat{\infty}$.

\section{The Optimization Problem.}

Given a finite b-complementary multisemigroup $A$ and a subset $M$ of $A$, the b-complementary multisemigroup problem defined as

$$
\begin{gathered}
\min \sum_{g \in M} c(g) t(g) \\
\text { s.t: } b \in \widehat{\sum_{g \in M} t} t(g) g \\
t \in \mathbf{N}^{M}
\end{gathered}
$$

where $c(g) \in \mathrm{R}$ for all $g \in M$.

The function $\pi: A \rightarrow R$ has subadditivity (see [2]) if it satisfies:

(i) $\pi(\emptyset)=-\infty$;

(ii) $\pi(G)=\max \{\pi(g): g \in G\}$ for all $G \subseteq A$;

(iii) $\pi(\{\widehat{0}\})=0$;

(iv) $\pi(G)+\pi(H) \geq \pi(G \widehat{+} H)$ for all $G, H \subseteq A$.

The Subadditivity Cone is the set

$$
C(A)=\left\{\left(\pi(g) ; g \in A_{+}\right): \pi \text { is a subadditivity function }\right\}
$$

We denote the linearity of $C(A)$ by $L(A), \pi(\{g\})$ by $\pi(g)$ and $(L, E)$ as a base of $C(A)$ (see $[6])$. 


\section{Duality}

Consider the following linear programming problem

$$
\begin{array}{r}
\min \widetilde{c} x \\
\text { s.t: } \widetilde{A} x=\widetilde{b} \\
\widetilde{E} x \geq \widetilde{h} \\
x \geq 0
\end{array}
$$

where $x$ and $c$ are an $n$ vetor, $\widetilde{b}$ is an $m$ vetor, $\widetilde{h}$ is an $p$ vetor, $A$ is an $m$ by $n$ matrix and $E$ is a $p$ by $n$ matrix. Corresponding to this problem, called primal problem, consider the following linear problem

$$
\begin{array}{r}
\max \tilde{\pi} \widetilde{b}+\widetilde{\mu} \widetilde{h} \\
\text { s.t: } \widetilde{\pi} \widetilde{A}+\widetilde{\mu} \widetilde{E} \leq \widetilde{c} \\
\widetilde{\pi} \text { unrestricted, and } \widetilde{\mu} \geq 0
\end{array}
$$

where $\widetilde{\pi}$ and $\widetilde{\mu}$ are row vetor of size $m$ and $p$, respectively. This problem is called the dual problem of the primal problem (1)-(4) (see 2.5 in [7]).

Now, for $(A, \widehat{+})$ be a b-complementary multisemigroup, let $P(A, b)$ the convex hull of the set $\left\{t \in \mathrm{N}^{A_{+}}: b \in \widehat{\sum}_{g \in A_{+}} t(g) g\right\}$. We denote by $P$ the following linear programming problem

$$
\begin{aligned}
& \min \sum_{g \in A_{+}} c(g) t(g) \\
& \text { s.t: } t \in P(A, b)
\end{aligned}
$$

where $c(g) \in \mathrm{R}$ for all $g \in A_{+}$.

In ([6]) Araoz and Johnson show the following theorem:

Theorem 4.1 [2, Theorem 3.8] Let $(L, E)$ be a base of $C(A)$. The following system defined a $P(A, b)$

$$
\begin{array}{r}
\sum_{g \in A_{+}} \rho(g) t(g)=\rho(b), \text { for all } \rho \in L \\
\sum_{g \in A_{+}} \pi(g) t(g) \geq \pi(b), \text { for all } \pi \in E \\
t(g) \geq 0, \text { for all } g \in A_{+} .
\end{array}
$$

In order to defined the dual problem of the problem $P$ we shown the following theorem. 
Theorem 4.2 The $P$ problem is equivalent to the $P_{p}$ problem

$$
\begin{aligned}
& \min \sum_{g \in A_{+}} c(g) t(g) \\
& \sum_{g \in A_{+}} \rho(g) t(g)=\rho(b), \quad \rho \in L ; \\
& \sum_{g \in A_{+}} \pi(g) t(g) \geq \pi(b), \quad \pi \in E ; \\
& t(g) \geq 0, \quad g \in A_{+},
\end{aligned}
$$

where $(L, E)$ is a base for $C(A)$ and $c \in R^{A_{+}}$

Proof. By theorem 4.1 the system (8)-(10) defined a $P(A, b)$, then the problem $P$ and $P_{p}$ are equivalent. $\diamond$

Theorem 4.3 The dual problem of $P_{p}$ is the problem $P_{d}$

$$
\begin{gathered}
\max \sum_{\rho \in L} \rho(b) v(\rho)+\sum_{\pi \in E} \pi(b) w(\pi) \\
\sum_{\rho \in L} \rho(g) v(\rho)+\sum_{\pi \in E} \pi(g) w(\pi) \leq c(g), \quad g \in A_{+} \\
v(\rho) \text { unrestricted, } \rho \in L \\
w(\pi) \geq 0, \pi \in E .
\end{gathered}
$$

Proof. Since $L$ and $E$ are finite sets (see [6]), the system (12)-(14) is the form of the system (2)-(4) where $n=\left|A_{+}\right|, m=|L|, p=|E|,(\widetilde{A})_{\rho, g}=\rho(g)$ for $\rho \in L, g \in A_{+},(\widetilde{E})_{\pi, g}=\pi(g)$ for $\rho \in E, g \in A_{+}, \widetilde{b}=(\rho(b): \rho \in L), \widetilde{h}=(\pi(b)$ : $\pi \in E)$ and $\widetilde{c}=\left(c(g): g \in A_{+}\right)$. Therefore the dual problem of $P_{p}$ is the form by duality linear programming the proof is complete $\diamond$

\section{References}

[1] Aráoz J. and Johnson E., "Polyhedra of Multivalued System Problems", Report No.82229-OR, Institut für Ökonometrie und Operations Research, Bonn, W. Germany (1982)

[2] Aráoz J. and Johnson E.," Morphic Liftings between of pairs of Integer Polyhedra",Research Report 89616OR, Int. Operations Research, Bonn 1989.

[3] Gastou G., "On facets of integer Programming Polyhedra", Ph.D. Thesis, Administrative Science Dept, Yale University, New Haven, 1982.

[4] Gomory R., "Some Polyhedra Related to Combinatorial Problems", Journal of Linear Algebra and Its Applications, 2(4),451-558 (1969). 
[5] Johnson E.L.,"Facets, Subadditivity, and Duality for Group and Semi-group Problems", Society for Industrial and Applied Mathematics 19103, Philadelphia,USA 1980.

[6] Rockafellar R. T., "Convex Analysis", Princenton Univerty Press, Princenton, New Jersey, U.S.A (1969)

[7] Salkin H. and Mat., "Foundations of Integer Programming", Elsevier Science Publishing, North-Holland (1989) 